\title{
Incompleteness and retrieval of case notes in a case note audit of colorectal cancer
}

\author{
Neil Vickers, Allyson Pollock
}

\begin{abstract}
Hospital case notes are a crucial source of data but are subject to two major biases: incompleteness of data and non-retrieval. To assess these biases in relation to colorectal cancer a study was performed of all cases of colorectal cancer listed in the Thames cancer registry in patients resident in one of four districts in South Thames regions with a diagnosis in 1988 . Five medical record sites were involved. Retrieval rate for all case notes for districts combined was $80 \%$. In two districts the rates were too high for further investigation; in the other two respectively patient survival and whether treatment was given were positively associated with retrieval. Among the four districts incompleteness of notes ranged from $38 \%$ to $62 \%$ for staging, $8 \%$ to $\mathbf{4 0} \%$ for treatment, and $70 \%$ to $25 \%$ for diagnostic tests. Information about treatment was missing in $3 \%$ to $20 \%$; survival data were omitted in less than $5 \%$. In all districts completeness of case notes was inadequate and in some nonretrieval compounded the problem. Missing data reduce the quality of cancer registry data and potentially undermine interpretation of epidemiological studies and evaluation of care. Further research is warranted into the standards and resourcing of medical records departments and their effects on retrieval and data quality. Structured proformas could be applied across specialties to identify missing items in case notes, to identify areas where standards are required, or to audit notes where standards have already been agreed. A staging protocol to set standards for colorectal cancer has been adopted in one district, and a prospective audit is being established.

(Quality in Health Care 1993;2:170-174)
\end{abstract}

Department of Public Health Sciences, $\mathbf{S t}$ George's Hospital Medical School London SW17 0RE Neil Vickers, research assistant

Allyson Pollock, senior lecturer

Correspondence to: Dr Pollock

Accepted for publication 9 July 1993 is widely assumed that the process of medical audit itself helps to increase the rates of

\section{Introduction} most important source of the audit cycle. Audit based on case notes is threatened by two main sources of bias: completeness and retrieval, ${ }^{12}$ comparatively few studies have attempted to quantify these changes and those that have done so have yielded conflicting results. ${ }^{3-6}$ Gabbay et al appraised the quality of two sets of case notes and concentrated on qualitative factors such as whether the notes were generally legible, whether the history of the presenting symptom was well recorded, and whether the initial management was appropriate ${ }^{3}$ - that is, the clinicians completing the questionnaire were asked to make a high proportion of value judgments. Not surprisingly, perhaps, interobserver variation was high. Rai et al in a study of records of geriatric patients concentrated on specific data items (for example, falls, incontinence, rectal examination, mobility) and found that the audit process did not improve the quality of notes kept, although their standard improved significantly when a standardised proforma was inserted into the notes as an aide mémoire to the clinicians. ${ }^{5} 6$

The extent of incompleteness is subject to bias from non-retrieval. Gulliford et al examined the impact of non-retrieval of case notes on medical audit and found lower retrieval rates for deceased patients and patients treated at teaching hospitals. ${ }^{7}$ Krarup and Lamont also recorded high non-retrieval rates in patients receiving certain diagnostic procedures. $^{8}$

This study examines both of these two possible biases in obtaining information: quality of information recorded and case note retrieval in four districts.

\section{Methods}

In 1990 we undertook an ecological analysis of all cases of colorectal cancer registered by the Thames Cancer Registry as diagnosed between 1982 and 1988 and showed significant variations in five year relative survival rates for residents of the 28 districts in the two South Thames regions (A Pollock, N Vickers, personal communication). Most data for the Thames Cancer Registry are collected by cancer registry clerks, who visit individual hospitals and treatment centres and retrieve data directly from case notes on to a structured proforma. These data are later entered on to the registry computer centrally. The remainder of the data come from death certificate notices supplied by the Office of Population Censuses and Surveys (OPCS). 
Because survival may be affected by the ability of cancer registry clerks to retrieve information on date of diagnosis we undertook a retrospective case note study in the four districts with the worst and best five year relative survival rates between 1982 and 1988 to investigate underlying reasons for differences in survival. This study forms part of a larger study examining biases in obtaining information from the cancer register and their impact on survival.

We looked at case notes of all patients recorded in the Thames Cancer Registry as having a diagnosis of colorectal cancer in 1988 who resided in the four districts (A-D), as defined above. Using cancer registry data, we established that most patients are diagnosed and treated in their district of residence $(83 \%$, $88 \%, 73 \%$, and $79 \%$ in districts A-D respectively). Because we did not have the resources to follow up a small number of cases at a large number of treatment centres we requested case notes only for patients with diagnosis or treatment in their district of residence. We also requested notes for patients whose district of diagnosis and treatment was not specified in the registry ( 38 patients) from the hospitals in their district of residence. Our total sample was 368 case notes.

The approval of the ethics committee was granted at the outset of the study. A fee was agreed for retrieval of case notes, and lists of cases were sent out to the senior medical records clerks. There were five medical record sites covering six hospitals and outlying outpatient departments in the four districts. We had hoped to retrieve notes within three months, but in some districts retrieval continued for 18 months, requiring repeat visits, telephone calls, and extra help from audit coordinators, local research assistants, and clinicians.

A structured proforma was used to extract the data and was devised after consultation with senior pathologists and clinicians and cancer registry staff, who defined their criteria for data collection. Each set of case notes was checked by two doctors: a senior registrar and a senior house officer in public health medicine.

\section{INCOMPLETENESS OF CASE NOTES}

Data items extracted from those case notes which were retrieved included demographic details, diagnostic investigations, details of the tumour and stage, treatment, and outcome (table 1). These correspond to data items collected by the cancer registry.

Stage is a measure of the severity of the disease and is important because it determines treatment and outcome. Two measurements of Dukes' stage were made. Dukes' stage "as indicated" refers to whether a Dukes' stage is recorded in the notes. Dukes' stage "as reconstructed" means reconstructed on the basis of clinical and histological data (on tumour, nodes, and metastasis). When there was no indication that the liver had been examined, either in the course of surgery or in a diagnostic investigation, Dukes' stage was taken as unreconstructable. The same stricture was applied to lymph node involvement: when unspecified, Dukes' stage was also deemed to be unreconstructable.

It was not always possible to ascertain whether a treatment or test had been given. When the notes neither mentioned requesting an investigation or treatment nor recorded whether it had occurred then the investigation or treatment was recorded as not done. However, if an investigation or treatment was recorded as having been requested or decided but neither treatment notes nor results of investigations were recorded, then the data item was considered as missing.

The analysis recorded the frequency of missing data items - that is, when data which should have been recorded were absent.

NON-RETRIEVAL

Gulliford et al ascertained that survival and treatment were associated with case note retrieval. To establish whether these factors persisted at district level, we repeated their methodology ${ }^{7}$ treating case note retrieval as a binomial trial and using some of their explanatory variables. The independent variables selected from the cancer registry included patient survival (alive or dead), district of residence, sex, whether the patient had received treatment or not, and whether the place of treatment was definitely known. Treatment included surgery, radiotherapy, and oncology but not palliative care. The associations of explanatory variables with retrieval were estimated by means of a logistic regression model, and the results are presented as odds ratios. The significance of associations was measured using the deviance difference as an approximate $\chi^{2}$ statistic.

\section{Results}

INCOMPLETENESS OF CASE NOTES

The figure depicts the range of missing data items from the case notes (shown as the bar), each district being ranked in order from left to right. In all four districts data on tumour morphology and stage were most frequently absent from case notes (up to $60 \%$ or so of

Table 1 Data items extracted from hospital records

\begin{tabular}{l}
\hline Item \\
\hline Demographic: \\
District of residence \\
Sex \\
Tumour: \\
Site \\
Morphology \\
Degree of differentiation \\
Diagnostic investigations: \\
Colonoscopy/sigmoidoscopy \\
Barium enema \\
Chest $x$ ray examination \\
Ultrasonography/computed tomography of liver \\
Treatments: \\
Surgery: \\
Type of operation \\
Emergency or elective \\
Chemotherapy \\
Radiotherapy \\
Outcome: \\
Date of death \\
\hline
\end{tabular}




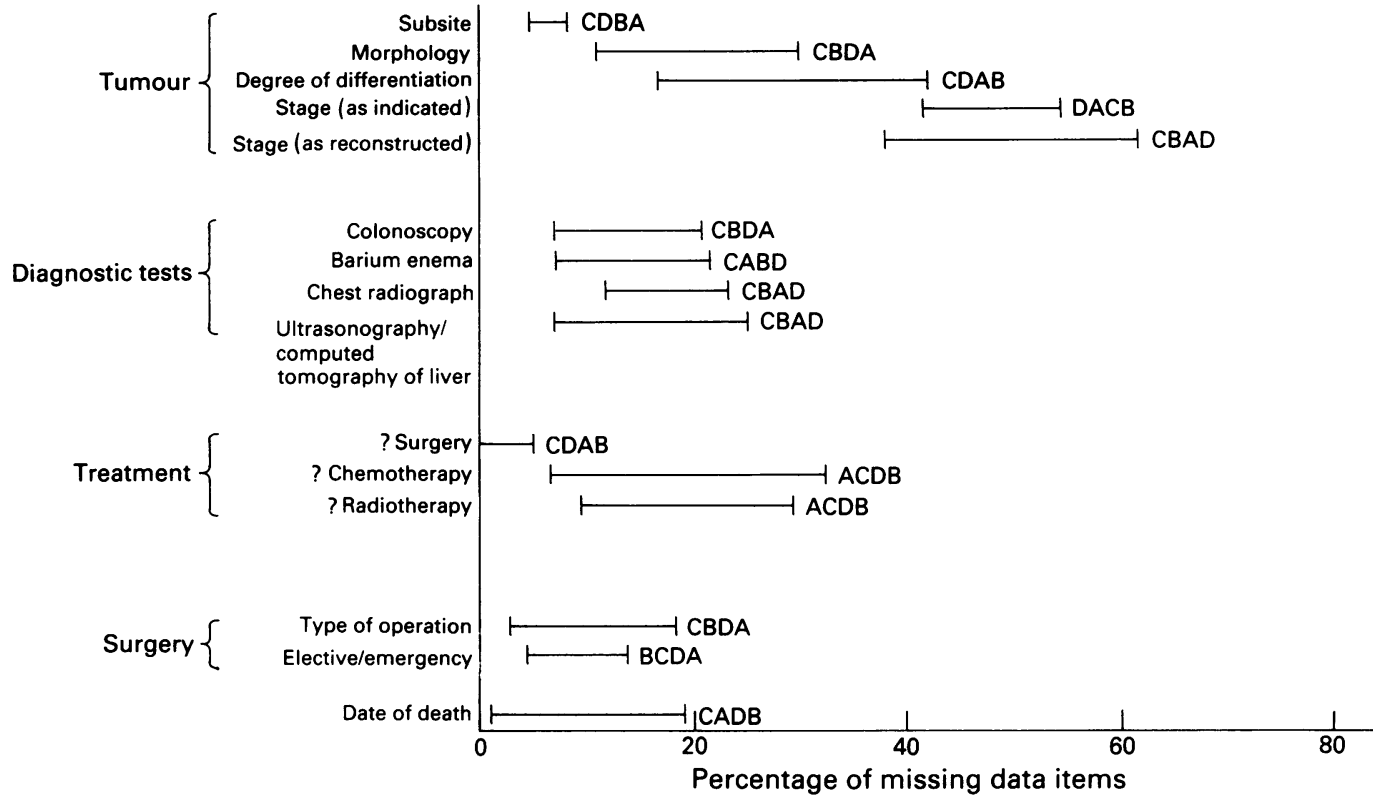

Incompleteness of data items in case notes $(n=368)$ of patients with colorectal cancer diagnosed in 1988 across four districts ( $A-D)$ in South Thames regions (bar shows range across districts)

cases). In $5 \%$ to $21 \%$ of cases it was unclear whether specified diagnostic investigations took place or not. In around 3\% of cases it is unclear whether surgery took place and in $18 \%$ whether chemotherapy and radiotherapy were performed. Where chemotherapy was recorded as having been given the proportions of patients receiving it in the four districts were $6 \%, 15 \%, 8 \%$, and $18 \%$ respectively. Among 195 patients known to have died, the date of death was absent in their case notes in only eight - that is, $4 \%$ (range $2 \%$ to $18 \%$ across individual districts).

NON-RETRIEVAL

The retrieval rate for all four districts combined was $80 \%(92 \%, 96 \%, 53 \%$, and $71 \%$ in districts $\mathrm{A}-\mathrm{D}$ respectively), and district of residence and case note retrieval were strongly associated $(\mathrm{p}<0.0001)$. In districts $A$ and $B$ the high rates of retrieval $(92 \%$ and $96 \%$ respectively) reduced the power of the study, which meant that we were unable to investigate factors related to non-retrieval. Table 2 shows the odds ratios of retrieval for districts $\mathrm{C}$ and $\mathrm{D}$. In district $\mathrm{C}$ alone patient survival was significantly and positively associated with retrieval of case notes, whereas in district $\mathrm{D}$ the only significant positive association with retrieval was whether patients had received treatment.

\section{Discussion}

INCOMPLETENESS OF CASE NOTES

The results of this study have serious implications for epidemiological and audit studies. Missing data on tumour morphology and degree of differentiation of the order of $20 \%$ will confound interpretation of any changes in the epidemiology of colorectal cancer. Similarly, though there are published reports of changes in the subsite distribution of colon cancer these rely on cancer registry data which have not been validated against case notes.
The omissions in data on tumour staging are also very serious. Dukes' stage, as the measure of the severity of a cancer, determines treatment and prognosis. Without adequate data on staging it is impossible to evaluate the effectiveness of care and also to interpret variations in treatment and survival rates across districts. Though it is not always possible to ascertain Dukes' stage on every case, standards could be set as to what proportion of tumours should be staged. Among patients who received surgical treatment, we found that the proportion of tumours staged varied dramatically $57 \%$ in district $\mathrm{A}, 46 \%$ in district $\mathrm{B}, 64 \%$ in district $\mathrm{C}$, and $74 \%$ in district $D)$. We suggest that a target be set for recording tumour stage in

Table $2 A$ logistic regression model of case note retrieval in Districts $C$ and $D$

\begin{tabular}{|c|c|c|c|}
\hline Variable & $\begin{array}{l}\text { Notes } \\
\text { retrieved } \\
(\%)\end{array}$ & $\begin{array}{c}\text { Odds ratio } \\
\text { (95\% confidence } \\
\text { interval) of retrieval }\end{array}$ & p Value \\
\hline \multicolumn{4}{|c|}{ District $C(n=73)$} \\
\hline $\begin{array}{l}\text { Survival: } \\
\text { Alive } \\
\text { Dead }\end{array}$ & $\begin{array}{l}79 \cdot 31 \\
36 \cdot 36\end{array}$ & $3 \cdot 99(1 \cdot 21$ to $13 \cdot 15)$ & $<0.05$ \\
\hline $\begin{array}{l}\text { Sex: } \\
\quad \text { Male } \\
\text { Female }\end{array}$ & $\begin{array}{l}41 \cdot 94 \\
61.90\end{array}$ & $0.41(0.14$ to $1 \cdot 24)$ & NS \\
\hline $\begin{array}{l}\text { Treatment } \\
\text { Yes } \\
\text { No }\end{array}$ & $\begin{array}{l}66 \cdot 04 \\
20 \cdot 00\end{array}$ & $3 \cdot 86(0.61$ to $24 \cdot 28)$ & NS \\
\hline $\begin{array}{l}\text { Patient de } \\
\text { Yes } \\
\text { No }\end{array}$ & $\begin{array}{l}\text { treated i } \\
55.07 \\
25.00\end{array}$ & $\begin{array}{l}\text { listrict } C: \\
1.93(0.09 \text { to } 43.88)\end{array}$ & NS \\
\hline \multicolumn{4}{|c|}{ District $D(n=68)$} \\
\hline $\begin{array}{l}\text { Survival: } \\
\text { Alive } \\
\text { Dead }\end{array}$ & $\begin{array}{l}80 \cdot 00 \\
68 \cdot 57\end{array}$ & $1.43(0.21$ to 9.56$)$ & NS \\
\hline $\begin{array}{l}\text { Sex: } \\
\quad \text { Male } \\
\text { Female }\end{array}$ & $\begin{array}{l}75 \cdot 76 \\
65 \cdot 71\end{array}$ & $1 \cdot 31(0.41$ to $4 \cdot 14)$ & NS \\
\hline $\begin{array}{l}\text { Treatment } \\
\text { Yes } \\
\text { No }\end{array}$ & $\begin{array}{l}81 \cdot 25 \\
61 \cdot 11\end{array}$ & $8.02(1.16$ to $55 \cdot 39)$ & $<0.02$ \\
\hline $\begin{array}{l}\text { Patient de } \\
\text { Yes } \\
\text { No }\end{array}$ & $\begin{array}{l}\text { treated i } \\
75.93 \\
50.00\end{array}$ & $\begin{array}{l}\text { listrict D: } \\
\quad 1.42(0.84 \text { to } 4 \cdot 14)\end{array}$ & NS \\
\hline
\end{tabular}


patients receiving treatment; similarly, standards could be set for investigations.

On the whole, treatment data were well recorded in the notes. Radiotherapy and chemotherapy are relatively rare forms of treatment in colorectal cancer, accounting for less than $15 \%$ of all treatments. In our discussions with clinicians, they indicated that incomplete data on chemotherapy and radiotherapy may often be owing to separate sets of notes being compiled for those treatments. This is problematic for cancer registry clerks attempting to retrieve data and may also have implications for patient care. In some cancers in which radiotherapy and chemotherapy may form a greater proportion of treatment the failure to retrieve information on these treatments, with their subsequent underestimation, could have serious implications for resource estimates. The variations observed across districts in chemotherapy and radiotherapy may be due to selection bias in retrieval of case notes or incompleteness of data.

The high degree of completeness for date of death probably indicates that most patients with colorectal cancer die in hospital, which we verified against data in the death certificate.

NON-RETRIEVAL

This study shows that retrieval rates in the four districts vary by district of residence and that the selective retrieval of case notes could bias the results of audit in some districts. This will be of concern to those involved in studies across districts or in regional audit, since biases in the retrieval of case notes may selectively influence the recording of information and make comparisons across districts difficult. This is illustrated in districts $\mathrm{C}$ and $\mathrm{D}$ where retrieved case notes were more likely to be of living patients or those who had received treatment. The different retrieval rates may be partly explained by our observations of the different arrangements and resources available for storing medical records. In district A notes are retained for 10 years after the last date of the patient's attendance or six years if the patient has died. Although the notes are stored at several sites and not on microfiche, they can be accessed more or less immediately. In district $\mathbf{B}$ all case notes are stored on a single site for the first two years after the last date of attendance; thereafter, they may be transferred to other sites. In district $B$ we observed an efficient and well resourced modern medical records department. Case note retrieval in district $\mathrm{C}$ is mainly from microfiche copies. Since records of surviving patients take priority over those of deceased patients in transfer to microfiche this could account for the survival bias observed. In district $D$, notes are transferred to microfiche only five years after the last date of attendance, and so most of the notes we requested should have been available in hard copy. However, we found that the methods of storing case notes of deceased patients and patients who have been discharged varied considerably across districts, and in districts C and D there was no systematic filing and storage of these case notes. This could account for the finding that survival and treatment were both positively associated with retrieval and also explains the difficulty in retrieving the case notes.

\section{OUTCOME OF THIS STUDY}

We presented our work to the local audit coordinators and clinicians in all four district hospital sites either at combined clinical and pathology meetings or at their postgraduate meetings. Purchasers attended some of these meetings. On two hospital sites we used case notes where data were absent or disagreed with our proforma to verify the process and illustrate the problems. We offered audit coordinators the opportunity to build on our work and disseminated the King's Fund consensus statement on colorectal cancer ${ }^{9}$ together with guidelines of the United Kingdom Coordinating Committee for Cancer (UKCCR) on staging for colorectal cancer, ${ }^{10}$ which many surgeons and pathologists had not previously seen. The initial response to our presentations varied, with some occasional hostility, but many clinicians readily admitted that they had no protocols for investigating and treating colorectal cancer. One surgeon said that staging was not important for outcome.

Several months after our presentation a senior pathologist who is chair of medical audit in one of the districts informed us that the pathologists and clinicians had agreed to use the UKCCR staging protocol to set standards for colorectal cancer but expressed concern that the standards might be too high for their current resources; we suggested their amendment in the light of expert opinion and local resources. The same district is taking forward a prospective audit of its case notes. Most of the other district hospitals have begun to develop standards for staging and treatment protocols.

In conclusion, this study shows that data on colorectal cancer in case notes are inadequate and that non-retrieval of case notes may further confound the interpretation of incomplete data in case note audit. There is no published account of the different standards and resourcing of medical record departments and this requires further study if medical audit committees are to address the issue of nonretrieval of case notes.

Missing data items will affect national cancer registry data and, in particular, the interpretation of epidemiological trends in incidence, treatment, and survival. Both regionally, and locally, missing data items will affect the evaluation of care. More work and closer liaison is required between medical audit officers, clinicians, and those who gather data, whether for purchasing or for other databases such as the cancer registry.

The methodology used in this study could be widely applied across specialties and treatment centres. Its advantages are threefold: it will allow clinicians to identify areas where standards are needed; it can be used to 
set standards, and it can help to monitor whether standards are being adhered to.

To address the problem of incompleteness clinicians could develop a proforma and audit its effects on completeness of case notes. Use of a proforma would have two major benefits: it would serve as an aid to developing clinical protocols and it would help cancer registry clerks to improve the completeness of their registers, making these better tools for epidemiological and public health related studies.

We thank the Thames cancer registry for providing data, D Rosalind Benster for her considerable help in retrieving case note data, Dr Mark McCarthy for resourcing the project, and South West Thames regional medical audit program for continuing support.
1 Heath DA. Random review of hospital patient records BMF 1990;300:651-2.

2 Shaw C. Medical audit: a hospital handbook. 3rd ed. London: King's Fund Centre, 1990.

3 Gabbay J, McNicol MC, Spiby J, Davies S. What did audit medical audit. $B M \mathcal{F} 1990 ; 300: 526-9$

4 Rai GS. Lessons from preliminary evaluation of a year's medical audit. $B M 7$ 1990;301:874-5.

5 Rai GS, Bielawska C, Sharland DE. A year's experience of auditing case notes. Care of the elderly 1991;3:291-2.

6 Rai GS, McInnes E, Phongsathorn V, Sharland DE. Medical audit of case notes on a one to one basis. $\mathcal{F} R$ Coll Physicians Lond 1991;25:358-9.

7 Gulliford MC, Petruckevitch A, Burney PG. Hospital case notes and medical audit: evaluation of non-response. $B M 7$ 1991;302:1128-9.

8 Krarup KC, Lamont AC. Hospital case notes and medical audit. BM7 1991;302:1605-6.

9 King's Fund Forum Consensus Statement. Cancer of the colon and rectum. London: King's Fund, June 1990

10 United Kingdom Coordinating Committee for Cancer. Handbook for the clinicopathological assessment and staging of colorectal cancer. London: UKCCR, 1990. 